\title{
Untersuchungen zur spät- und postglazialen Vegetationsgeschichte im nördlichen Bayern (Rhön, Grabfeld, Lange Berge)

\author{
JÜRGEN HAHNE
}

Institut für Palynologie und Quartärwissenschaften der Universität Gōttingen, BRD

\author{
Investigations on the Late- and Postglacial History of Vegetation of Northern Bavaria \\ (Rhön, Grabfeld, Lange Berge)
}

\begin{abstract}
Palynological investigations of sediments from northern Bavaria (Rhön, Grabfeld, Lange Berge) reveal the Late Glacial and Postglacial history of the regional vegetation.

The older sediments were found in the Rhön (Schwarzes Moor) and date back into the Bölling Period. At the end of that period pine spread into the Grabfeld. In both areas Lacher Tuff has been found. A radiocarbon date of $10,300 \mathrm{BP}$ was found for the Late Glacial - Postglacial transition and one of $9300 \mathrm{BP}$ for the Preboreal - Boreal transition. Hazel reached its highest values in the Rhön around 7,400 BP. During the Atlanticum a deciduous mixed oak forest covered the Rhön and Grabfeld regions. Beech dominated since the Subatlanticum. In the Lange Berge region, however, a mixed forest with Fagus, Picea, Pinus and Abies developed.

In the Rhön first anthropogenic influence was found during the Latène Period. The boundary between zone IX and $\mathrm{X}$ has been dated at $820 \mathrm{~A} . \mathrm{D}$, and the start of extensive forest clearances at $1000 \mathrm{~A} . \mathrm{D}$. A culmination of landuse was found for the Medieval Period. At the end of that period however the Rhön was deserted. New forest clearances started around 1500 A.D., but were interrupted by the "Thirty Years War". Afterwards the Rhön got its present appearance.
\end{abstract}

\section{Zusammenfassung}

Im nördlichen Bayern wurden in den Lanschaften Rhön, Grabfeld und Lange Berge 3 Moore pollenanalytisch und auf Großreste hin untersucht.

Die ältesten untersuchten Sedimente stammen aus der Rhön und wurden der Böllingzeit (Zone Ib) zugeordnet. Dieser Abschnitt konnte in eine ältere Strauchphase und einen jüngeren Abschnitt mit verstärkter Ausbreitung von Baumbirken untergliedert werden. Im klimatisch begünstigten Grabfeld ist am Ende von Ib mit einer Einwanderung der Kiefer zu rechnen. Außer in der Rhön wurde auch im östlich anschließenden Grabfeld der Laacher Tuff nachgewiesen. Für die Grenze Spätglazial/Postglazial wurde durch eine absolute Datierung ein Alter von $10300 \mathrm{BP}$ ermittelt. Die Grenze Präboreal/Boreal wurde auf 9000 BP datiert. Das boreale Hasel-Maximum fallt in der Rhön auf den Zeitraum um 7400 BP. Im Atlantikum entwickelten sich Rhön und Grabfeld zu reinen Laubwaldgebieten mit hohen EMW-Anteilen. Yom Subatlantikum an dominierte hier die Rotbuche, deren rationelle Grenze auf 4900 BP datiert wurde. In den Langen Bergen kommt es hingegen zu einer Ausbildung von Mischwäldern, in denen neben den EMW-Arten Fichte, Kiefer und wohl auch die Tanne vorherrschen.

Im Schwarzen Moor konnten viele siedlungsgeschichtlich relevante Vorgänge dargelegt werden: Danach zeichnet sich eine erste Besiedlung des Untersuchungsgebietes in der Latène-Zeit ab. Die Grenze IX/X wurde mit dem Anstieg der Getreidekurve auf $1 \%$ definiert und auf $820 \mathrm{u} . \mathrm{Z}$. datiert. Es konnte der Beginn einer ersten Rodungsphase um $1000 \mathrm{u} . Z$. und ein Höhepunkt der Besiedlung im Hochmittelalter nachgewiesen werden. Im späten Mittelalter zeichnet sich eine Wüstungsperiode ab, auf die ab 1500 eine erneute starke Rodungsphase folgt. Durch die Auswirkungen des Dreißigjährigen Krieges wird diese unterbrochen, und erst im AnschluB daran erhält die Rhön ihr heutiges Aussehen. 


\section{Einleitung}

Im nördlichen Bayern gehören das Grabfeld und die Langen Berge zu den Gebieten, von denen bisher vegetationsgeschichtliche Informationen fehlen. Von der Rhön veröffentlichte Overbeck (1928) die ersten Pollendiagramme (Rotes und Schwarzes Moor). Von OVERBECK \& GRIÉz (1954) folgte eine vegetationsgeschichtliche und moorkundliche Arbeit über das Rote Moor. OVERBECK (1962) ergänzte diese durch einige 14C-Datierungen. BEUG (1957) arbeitete über die spätglaziale Vegetationsgeschichte der Rhön am Beispiel des Roten Moores, und GAUHL (1990) untersuchte die Entwicklungsgeschichte des Schwarzen Moores. Eine vegetationsgeschichtliche und pflanzensoziologische Arbeit von STREITz (1984) über das westliche Vorland der Rhön bildet den bisherigen AbschluB dieser Reihe. Neue Untersuchungen waren vor allem für das Gebiet der Rhön wünschenswert.

Die vorliegenden Untersuchungen wurden in den Jahren 1983-j986 am Institut für Palynologie und Quartärwissenschaften der Universität Göttingen durchgeführt.

Ganz herzlichen Dank möchte ich meinem Lehrer, Herm Prof. Dr. H.-J. Beug sagen, der mich in die Pollen- und GroBrestanalyse einführte. Prof. BEug gab die Anregung zu dieser Arbeit und war bei allen auftretenden Problemen stets eine große Hilfe.

Herr Prof. Dr. M. A. GEYH (14C-Laboratorium des Niedersächsischen Landesamtes für Bodenforschung, Hannover) führte die absoluten Datierungen durch. Herr Prof. Dr. Juvigne (Universität Liege, Belgien) untersuchte das Tuffmaterial aus Rappershausen. Herr Prof. Dr. E. GrUGER half mir bei der Bestimmung von Grobresten. Herr Dr. H. Stalling unterstützte mich bei Geländearbeiten sowie bei der Bestimmung von Grobresten und Pollenformen. Frau U. JAsCHINSKI half mir bei der Anfertigung von Tabellen und Diagrammen. Ihnen allen sage ich an dieser Stelle meinen Dank. Gleichermaßen gilt mein Dank der Regierung von Unterfranken für die Erteilung einer Ausnahmegenehmigung zu Arbeiten in Naturschutzgebiet Schwarzes Moor.

\section{Das Untersuchungsgebiet}

Die Rhön gehört zum Komplex der hessisch-thüringischen Mittelgebirge. Auf ihrem aus Muschelkalk und Buntsandstein aufgebauten Sockel liegen besonders in Mulden seit dem jüngeren Tertiär Tone und Quarzsande. Diese wurden im Miozän ungleichmäßig von einer Basaltdecke überzogen. Durch Verwitterung besonders geringmächtiger Bereiche in der Basaltdecke traten fensterartig an einigen Stellen die wasserundurchlässigen Tone wieder an die Oberfläche und schufen gemeinsam mit Verwitterungsproduktion des Basalts gute Voraussetzungen für die Entstehung von Mooren (Mensching 1957).

Das Grabfeld ist eine mehr hügelige Landschaft auf einem Niveau yon $300-400 \mathrm{~m}$ NN. Im Norden wird sie vom Oberlauf der Werra, im Norwesten von der Hohen Rhön und im Südosten von den HaBbergen begrenzt. KNOCH (1952) stellt das Grabfeld im Rahmen einer naturräumlichen Gliederung in das Gebiet der Mainfränkischen Platten. Es ist vorwiegend aus Muschelkalk und Keuperschichten aufgebaut und trägt teilweise cine LöBabdekkung.

Das Gebiet der Langen Berge ist eine recht kleine geographische Einheit. Aufgebaut aus Buntsandstein, Keuper und teilweise auch aus Muschelkalk, grenzt es unmittelbar an den südlichen Teil des Thüringer Waldes. Im Süden reicht es bis an die Linie Rodach-Meeder-Coburg heran.

Das Klima des Untersuchungsgebietes läßt sich nach VoLIRATH (1957) als gemäligt und ozeanisch beeinfluBt mit kontinentaler Tönung charakterisieren. Die mittleren Jahresniederschläge erreichen auf den höchsten Erhebungen der Rhön nur wenig mehr als $1000 \mathrm{~mm}$. Verglichen mit dem stärker ozeanisch beeinflußten Harz, wo in Höhenlagen um $600 \mathrm{~m}$ NN bereits deutlich höhere Werte erreicht werden, können die Niederschläge nur als máßig hoch bezeichnet werden. Ausgesprochen geringe Niederschläge von nur $500-600 \mathrm{~mm}$ treten im Grabfeld auf, das im Regenschatten der Rhön liegt. Im Gebiet der Langen Berge ( $400-528 \mathrm{~m}$ NN) kommt es dagegen durch Wolkenstau am südwestlichen Rand des Thüringer Waldes zu überdurchschnittlich hohen Niederschlägen von 750-800 mm im Jahr. Die Jahresdurchschnittstemperatur liegt im Grabfeld und in den Langen Bergen bei $7-8^{\circ} \mathrm{C}$, in der Rhön bei $5-6^{\circ} \mathrm{C}$.

Einen Uberblick über die Vegetationsgliederung des Untersuchungsgebietes gibt SEIBERT (1968) auf seiner Karte der natürlichen potentiellen Vegetation von Bayern $(1: 500000)$, aus der in Abb. 2 die nördlichen Teile Unterfrankens vereinfacht entnommen wurden. Die Rhön ist danach ein Buchenwald-Gebiet. Die Basaltkuppen tragen einen Zahnwurz-Buchenwald (Cardamino bulbiferae-Fagetum), auf Keuper und Buntsandstein stockt ein Hainsimsen-Buchenwald (Luzulo-Fagetum). Auf den übrigen sauren bis mehr basischen Gesteinen sind der reine Platterbsen-Buchenwald (Lathyro-Fagetum typicum) und der Perlgras-Buchenwald (Melico-Fagetum) ausgebildet. 




Abb. 1. Lage des Untersuchungsgebietes. 1 Schwarzes Moor in der Rhön; 2 Moor bei Rappershausen im Grabfeld; 3 Moor bei Rottenbach in den Langen Bergen.

Nach Overbeck \& Griéz (1954) liegen die höchsten Buchenvorkommen heute bei $840 \mathrm{~m}$ NN. Die montanen Laubwälder beginnen bei $600 \mathrm{~m} \mathrm{NN}$. Hier sind, besonders in Talschluchten und an Hängen im Bereich der Blockhalden, auch Fraxinus excelsior, Acer pseudoplatanus, Acer platanoides, Tilia cordata, Tilia platyphyllos und Ulmus glabra verbreitet.

Das Grabfeld ist mit seinen Böden über Keuper, Muschelkalk und Löß ein Eichen-Hainbuchen-Gebjet (GalioCarpinetum, Galio-Carpinetum typicum und Galio-Carpinetum luzuletosum), Lediglich ein kleiner nordöstlicher Bereich (Keuper) wird als Winterlinden-Traubeneichen-Gebiet (Luzulo-Quercetum) bezeichnet. Im Bereich der Langen Berge wird der nordöstliche, auf etwas höherem Niveau gelegene Teil zum Buchenwald-Gebiet gestellt (auf Buntsandstein: Luzulo-Fagetum; auf Muschelkalk: Lathyro-Fagetum typicum). Der südwestlich anschließende Teil, der sich bis an die Linie Coburg-Sonnefeld erstreckt, ist ein Eichen-Hainbuchen-Gebiet.

\section{Siedlungsgeschichte}

Erste menschliche Spuren sind im Untersuchungsgebiet für das Paläolithikum nachgewiesen worden (MALTER 1967). Hinweise auf eine erste länger andauernde Besiedlung ergeben sich für den Zeitraum der süddeutschen Umenfelderkultur im 13.-8. Jahrhundert v.u.Z. (MOLlek-Karpe 1959). Aus der mittleren und späten Phase dieser Zeit sind nach HiLKE (1970) Siedlungsplätze $2 \mathrm{~km}$ westlich von Rottenbach in den Langen Bergen entdeckt worden. In die Hallstattzeit ( $800-400$ v.u.Z.) gehören 7 Gräber, die bei Großeibstadt (Grabfeld) gefunden wurden. Nach Kossack (1970) sind sie in den Zeitraum von 675-600 v.u.Z. zu stellen. Auch bei Bastheim am Fuße der Rhön wurden Gräber der ältesten Hallstatstufe nachgewiesen. Nekropolen gleicher Zeitstelhung finden sich im östlichen Rhönvorland, Hügelgräber am Unterlauf der Milz. In der Latènezeit $(400$ v.u.Z.-0) wurden auf zahlreichen Rhönbergen Fliehburgen angelegt, von denen die Ringwallanlage auf der Milseburg die bedeutendste war (VONDERAU 1926). Im Gebiet um Münnerstadt (Grabfeld) ergaben sich Hinweise auf Siedlungen während der späten römischen Kaiserzeit im 2.-4. Jahrhundert u. Z. Fïr das 4. und 5. Jahrhundert kann man im Grabfeld von

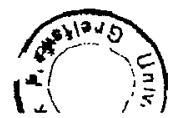




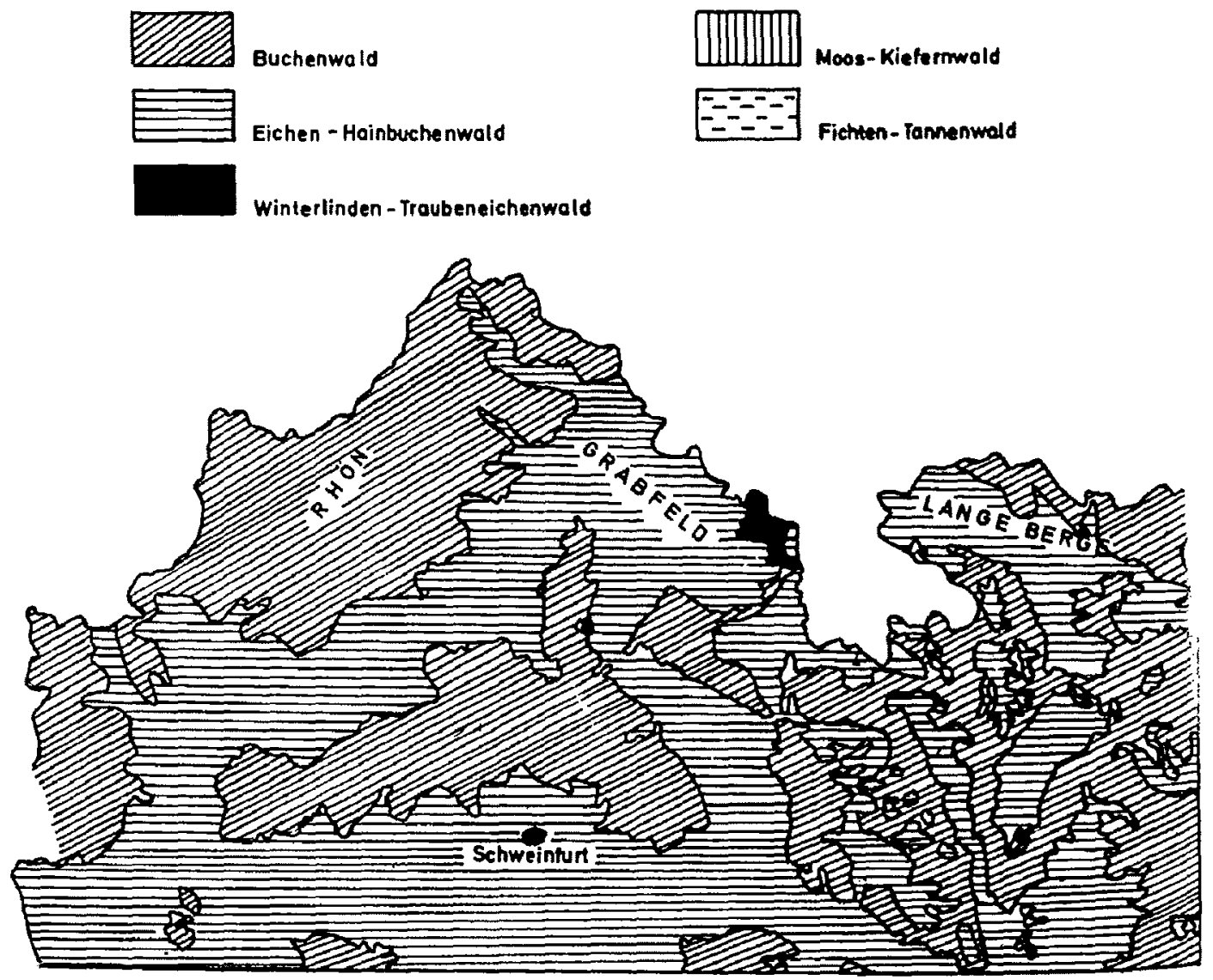

Abb. 2. Vegetationsgliederung des Untersuchungsgebietes. Verändert nach der Obersichtskarte der potentiellen natïrlichen Vegetationsgebiete von Bayem (1968) 1:500000.

einer Besiedelung durch Alemannen ausgehen (STUhLfauth 1962). Nach GuTtEnberg (1953/54) fülte sich das Grabfeld im 7. Jahrhundert nach und nach mit frănkischen Siedlern auf. Die Rhön wurde zur Zeit der Gründung des Klosters Fulda um 744 von 3 bedeutenden Handelswegen geschnitten, von denen einer, der als Ortesweg bezeichnet wird, unmittelbar am Roten Moor vorbeiführte. Im 9. Jahrhundert schritt die Besiedlung der Hochlagen voran. Bereits um 800 existierte in unmittelbarer Nähe des Schwarzen Moores eine Siedlung. Von den Temperaturen des Hochmittelalters begünstigt, setzte etwa ab 1000 neben weiter fortschreitender Siedlungstätigkeit auch eine verstärkte Rodungsphase ein. Im Coburger Gebiet (Lange Berge) wird um 900 zuerst Rodach urkundlich enwähnt (Malter 1965). Es folgen Tambach (11. Jahrhundert) und Coburg (11./12. Jahrhundert). Im 14. Jahrhundert werden erstmals Waldschutzverordnungen erlassen, um die stetig fortschreitende Entwaldung cinzudämmen. Bis zum Ende des 15. Jahrhunderts wird dann der gesamte nordbayerische Raum, bedingt durch Seuchen und eine Klimaverschlechterung, von einem deutlichen Siedlungsrückgang erfaßt. Nach einer erneuten Siedlungswelle ab 1500 werden zahlreiche Glashütten gegründet, durch die die Rhön noch stärker entwaldet wird. Der DreiBigjährige Krieg führt zu einer starken Entvölkerung der Rhön (OverBecK \& GRIÉz 1954). Im weiteren 17 ., aber auch noch im 18 . Jahrhundert wird dann das Abholzen der Wälder wieder fortgesetzt und damit das heutige Gesicht dieses Mittelgebirges geschaffen.

\section{Methodik}

Soweit möglich wurden alle Profile sowie das Material für 14C-Datierungen mit einem abgewandelten Modell des russischen Instorf-Bohrers (Russen-Bohrer) gewonnen. Bei größeren Torfmächtigkeiten muBten die ältesten Sedimente mit einer Dachnowski-Sonde erbohrt werden. Im Moor bei Rappershausen (Grabfeld) wurden die jüngsten Profilteile aus einem Carex elata-Bult mit einem Zinkblech-Kasten entnommen. 


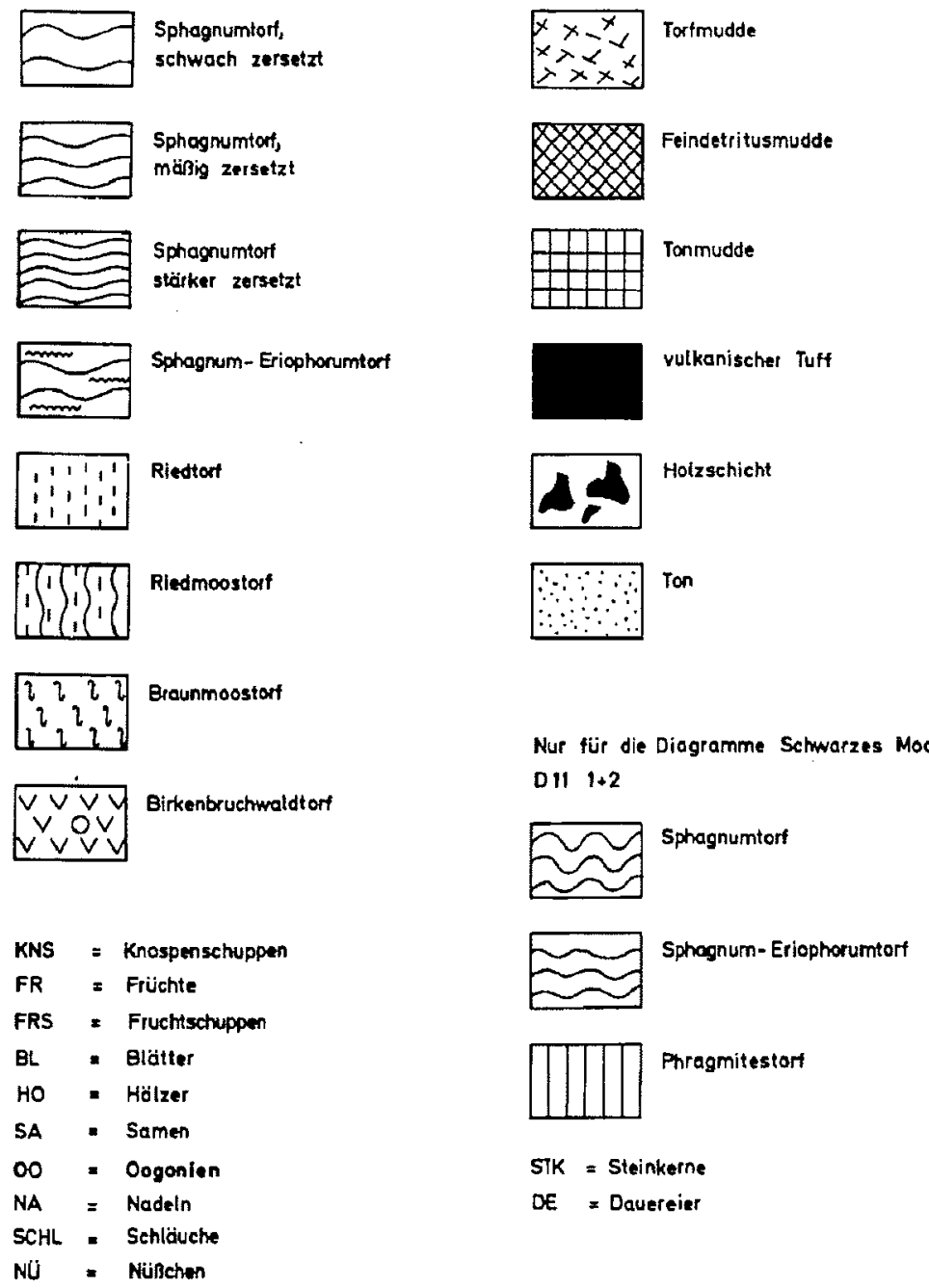

Abb. 3. Legende zu den Pollen- und Sporendiagrammen sowie zu den GroBresttabellen.

Die Aufbereitung der Pollenproben im Labor erfolgte nach der Methode von Bevg (1957). Proben mit silikatischen Bestandteilen wurden mit $70 \%$ iger HF behandelt und nach einer Acetolyse mit Hilfe von Ultraschall durch Absieben der Fraktion $<10 \mu \mathrm{m}$ gesäubert. Für GroBrestanalysen wurden die Bohrkerne in Stïcken von $10-20 \mathrm{~cm}$ Länge mit $5 \%$ iger $\mathrm{KOH}$ behandelt und nach Zerfall über Siebe mit 1 und $0,1 \mathrm{~mm}$ Maschenweite gegeben. Bis auf wenige spätglaziale Proben aus dem Schwarzen Moor wurden mindestens 500 Baumpollen (BP) ohne Corylus pro Probe ausgezählt. Da das Pollendiagramm vom Schwarzen Moor überwiegend spätglaziale Pollenspektren hat, wurde es als Gesamtdiagramm dargestellt, in dem BP und Nichtbaumpollen (NBP) unter Ausschluß von Farnen, Sumpf- und Wasserpflanzen, Sporen und umgelagerten PK eine Grundsumme von $100 \%$ bilden. Auf der rechten Seite dieses Diagramms wurde zusätzlich noch ein BP-Diagramm mit den wichtigsten Holzpflanzen-Sippen angefügt. Alle anderen Diagramme wurden als BP-Diagramme berechnet und dargestellt. Im Diagramm „Moor bei Rottenbach“ wurde Betula wegen überstarker lokaler Pollenproduktion aus der BP-Summe ausgeschlossen. Im Diagramm „Rappershausen B“ wurde dasselbe für Betula. Ainus, Salix und Rhamnus getan. 


\section{Die untersuchten Moore}

\subsection{Das Schwarze Moor in der Rhön}

Das Schwarze Moor liegt zwischen den Ortschaften Fladungen und Wüstensachsen in einer Mulde der Hochfläche östlich des Querenberges und in einer Höhe von 770-882 m NN (TK 25, Blatt 5426 Hilders, r: 3575880 , h: 5597700). Es nimmt hier die Wasserscheide zwischen Main und Fulda ein. Nach Nordwesten entwässert es durch den Birxer Graben, der in die Ulster mündet, nach Südosten in den Eisgraben, dessen Wasser über die Streu in die Fränkische Saale gelangt. Mit einer Nord-Süd-Ausdehnung von ca. $1100 \mathrm{~m}$ und einer Ost-West-Ausdehnung von $500 \mathrm{~m}$ im südlichen Teil hat das Moor nach REIMERs (1924) eine Größe von 55 ha. Rechnet man die randlichen Niedermoore noch hinzu, so beträgt die Gesamtfläche sogar 70 ha. Das Schwarze Moor wird von GAUHL (im Druck) als ein exzentrisches, ombrosoligenes Moor bezeichnet. Seine zahlreichen Kolke und Flarke geben ihm nach GIEs (1972) ein einmaliges Aussehen unter allen Mittelgebirgsmooren.

Der Beginn der Vermoorung setzte nach GauHL (im Druck) unmittelbar am Südrand des heutigen Hochmoorteils nördlich des Eisgrabens in der Älteren Tundrenzeit ein. Von hier aus verläuft in Nord-Süd-Richtung eine Rinne, in der die Vermoorung auf einer Breite von ca. $100 \mathrm{~m}$ hangaufwärts in nördlicher Richtung zungenförmig voranschritt. Am Ende des Präboreals hatte die Vermoorung etwa die Hälfte der heutigen Nord-Süd-Ausdehnung dieser Rinne erreicht.

\section{PROFILBESCHREIBUNGEN}

\section{Profil E 12 (Abb. 4 und Tab. 1)}

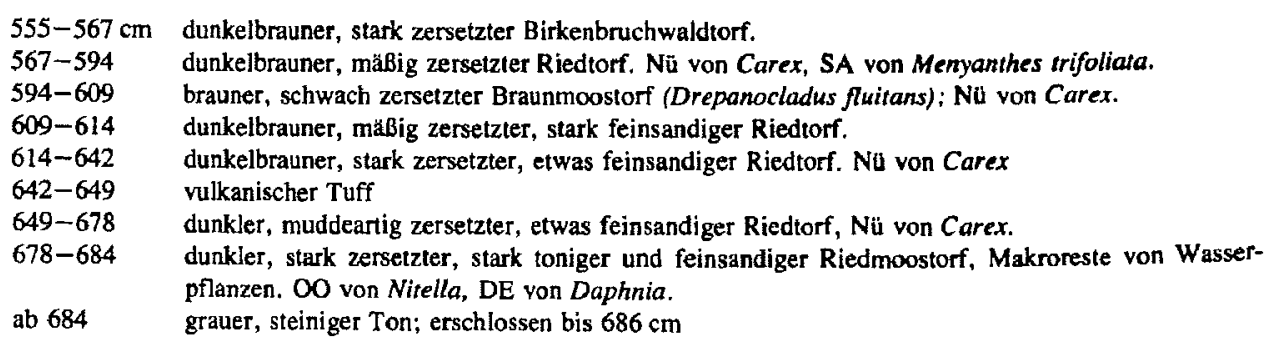

\section{Profil D 11 (Abb. 5 und 6)}

Bohrgerät: Russenbohrer

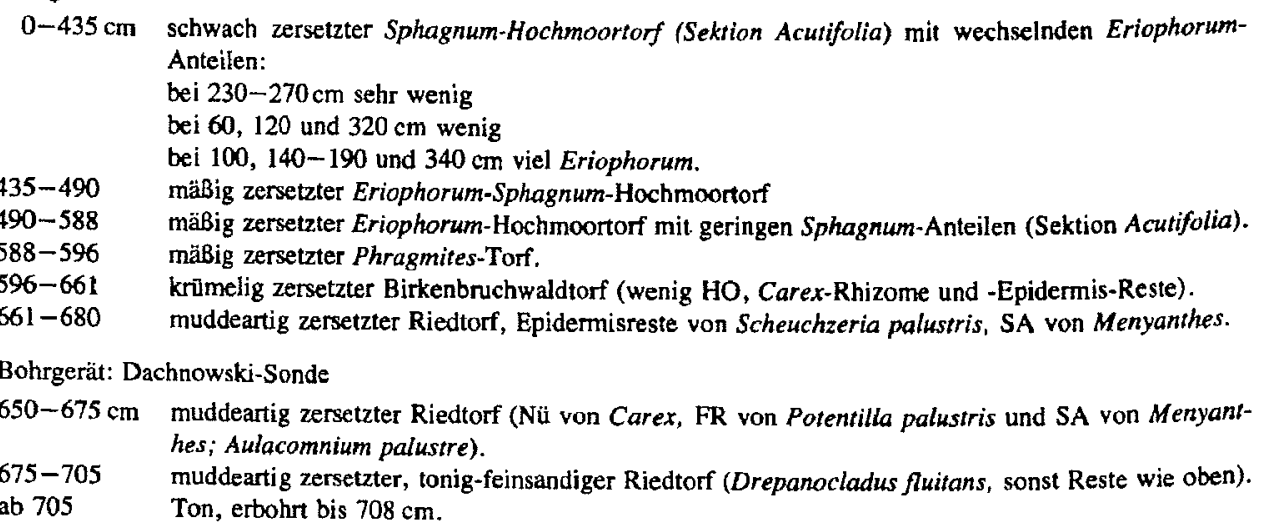


Tab. 1. GroBresttabelle Schwarzes Moor (Profil E 12).

Tob. 1: Schworzes Moor/Rhön - Grofreste

- In geringer Menge vorhanden .. holutilg


\subsection{Das Moor bei Rappershausen im Grabfeld}

Dieses Moor liegt $2 \mathrm{~km}$ westlich der Ortschaft Rappershausen, ca. $35 \mathrm{~km}$ südöstlich vom Schwarzen Moor. Es befindet sich zwischen den Flüssen Milz und Fränkische Saale bei $383 \mathrm{~m}$ NN (TK 25, Blatt 5628 Königshofen i. Grabfeld, r: 35989560 , h: 55834050). Der vermoorte Bereich ist weitgehend von Wald umgeben, und nur von Osten her reichen Äcker und Wiesen bis an seinen unmittelbaren Rand heran. Bei nahezu runder Form, mit einem Durchmesser von ca. $120 \mathrm{~m}$ in einem ebenen Gelände, könnte ein Erdfall die Voraussetzung für eine anschließende Vermoorung geschaffen haben. Das Profil wurde im zentralen Teil des Moores entnommen. Hier stocken größere Mengen von Betula pubescens, von denen derzeit viele im Absterben begriffen sind. Es gibt offene Wasserflächen und Schwingrasen. Verbreitet sind Großseggenbestände (Carex elata und $C$. rostrata).

\section{Stratigraphie (Abb. 7 und .8, Tab. 2)}

Für den oberen Bereich dieses Profils wurde ein Carex elata-Bult vertikal aufgesägt und ein Kastenprofil von $40 \mathrm{~cm}$ Länge entnommen.

$0-28 \mathrm{~cm} \quad$ unzersetzte und schwach zersetzte Carex-Rhizome und -Blattscheiden. Viele Nü und SCHL von Carex, viele FR und FRS von Betula pubescens, etwas Calliergon cuspidatum und Sphagnum (Sektion Cymbifolia).

28-40 brauner schwach zersetzter Riedtorf. Makroreste wie oben, jedoch SCHL von Carex meist zersetzt. Unterer Profilteil (Russenbohrer): 
$0-20 \mathrm{~cm}$ wässrig, in der Bohrkammer zerlaufend.

20-35 dunkelbrauner, stark zersetzter Birkenbruchwaldtorf, z.l. tonig.

35-275 brauner, mäBig zersetzter Birkenbnuchwaldtorf. Makroreste von Wasserpflanzen.

275-365 dunkelbrauner, stark zersetzter Birkenbruchwaldtorf. Bis $350 \mathrm{~cm}$ viel HO von Betula und Makroreste von Wasserpflanzen; ab $335 \mathrm{~cm}$ gröBere Moosanteile (Camplorhecium).

365-425 hellbrauner, schwach zersetzter Braunmoostorf; ab $416 \mathrm{~cm}$ mäBig zersetz. Makroreste von Befula. 425-429 Laacher Bimstuff.

429-508 hellbrauner, schwach zersetzter Braunmoostorf; bis $440 \mathrm{~cm}$ mäBig zersetza, ab 488 mit Feinsand durchsetzt. PK nur bis $491 \mathrm{~cm}$.

ab 508 grauer Ton; erschlossen bis $511 \mathrm{~cm}$.

\subsection{Das Moor bei Rottenbach (Lange Berge)}

Das Moor liegt ca. $14 \mathrm{~km}$ nordöstlich von Coburg und $1,5 \mathrm{~km}$ nordöstlich der Ortschaft Rottenbach in einem Gebiet, das im Norden von den Flüssen Werra und lltz begrenzt wird. Südliche Ausläufer des Thüringer Waldes reichen bis unmittelbar an das Gebiet heran (TK 25, Blatt 5631 Meeder, r: 4426260, h: 5584670).

Es handelt sich um ein kleinflächiges, in einer Mulde gelegenes Quell-Bruchmoor, dessen Zentrum von einer schmalen Quellrinne gebildet wird. Vorherrschende Arten sind hier Sphagnum riparium, Carex nigra und Molinia coerculea. An den angrenzenden nassen Moorteilen finden sich auch Sphagnum palustre, Polytrichum commune, Eriophorum latifolium und $E$. vaginatum. An der mäBig feuchten Peripherie hat sich ein dichter Baumbestand mit Betula pubescens, Rhamnus frangula und Picea abies, an trockeneren Stellen auch mit Pinus sylvestris angesiedelt. Das Profil wurde unmittelbar neben der Quellrinne entnommen.

\section{Stratigraphie (Abb, 9)}

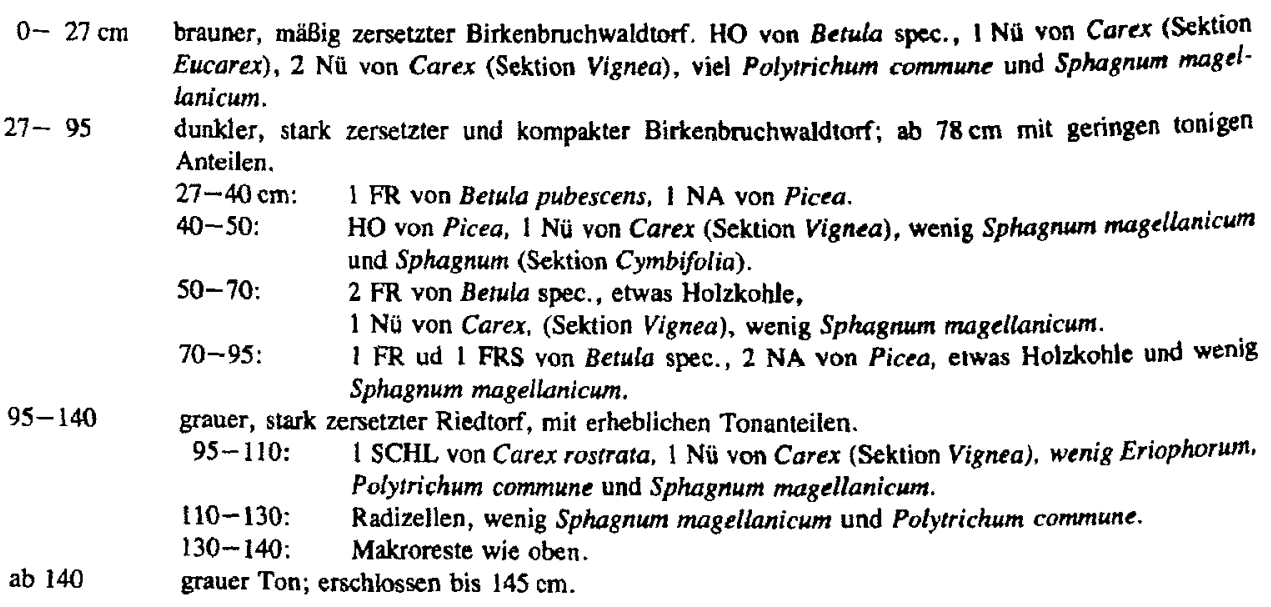

\section{Vegetationsentwicklung und Datierung}

\subsection{Späteiszeit}

\subsection{1. Ältere Tundrenzeit (Zone I)}

Die Schlußphase der Ältesten Tundrenzeit (Zone Ia) ist in den Pollendiagrammen aus den nördlichen Mittelgebirgen häufig durch den Rückgang der NBP-Anteile, besonders der Gräser, charakterisiert. Die ältesten Spektren aus dem Schwarzen Moor (Abb. 4) zeigen dieses nicht. Da sie aber prä-allerödzeitlich sein müssen (Laacher Tuffschicht im Hangenden) werden sie der Böllingzeit (Ib) zugeordnet. Aufgrund guter Übereinstimmung mit den Profilen „Großenmoor" 


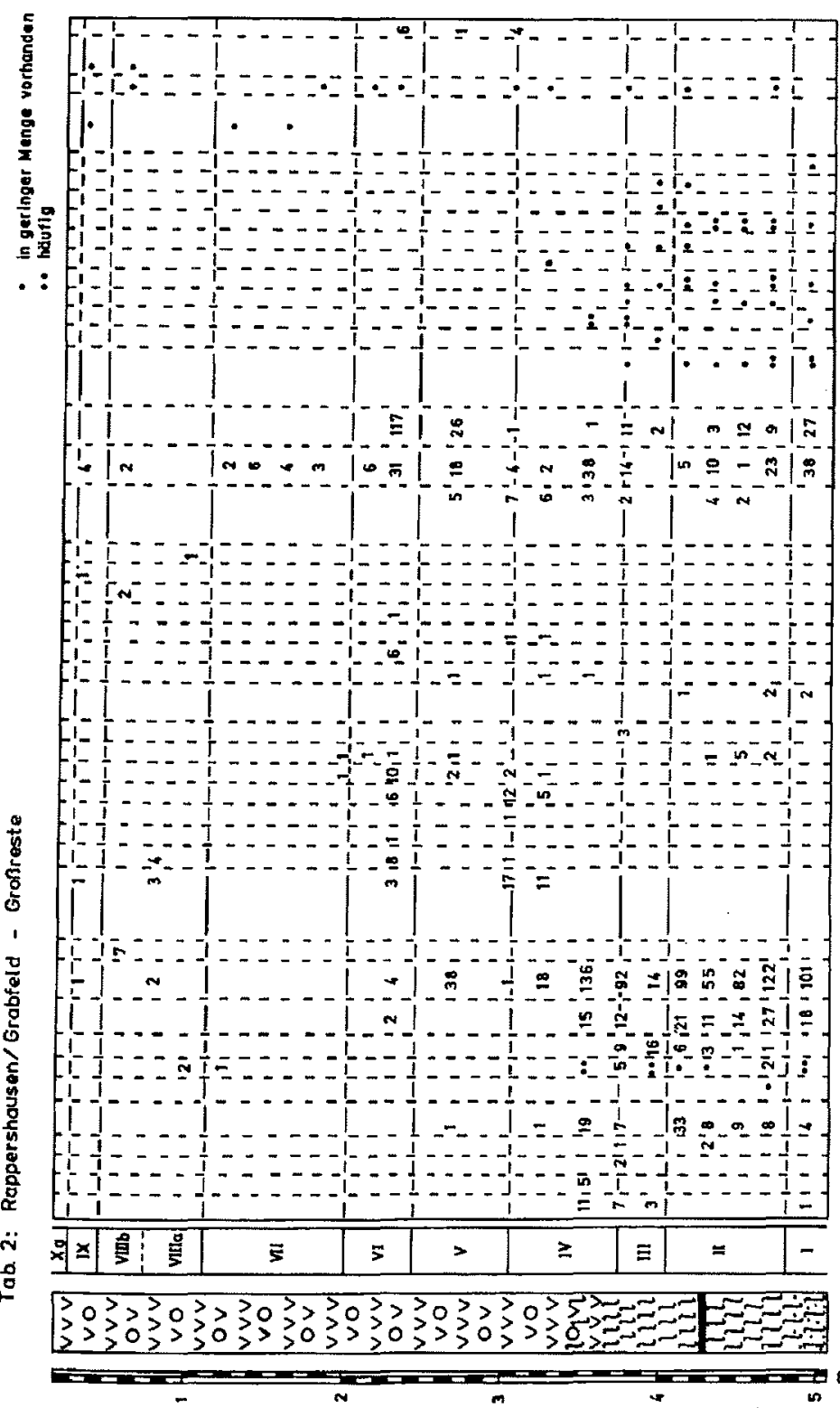

CHARA OO SPHAG: SEKT, CUSPIOAIA CYMBIFOLIA SPHAGNUM Magellanicum

THUIDIUM LANATUM SCORPIDIUM SCORPIDIOID. HYGROAM QYYSTEGIUM FLUV DRE PANOCLADUS VERNICOS. CINCLIDIUM FIYUGAS CHRYSOHYPNUM SIELLATUM CAMPTOTHECIUM NITENS CALLIERGON STRAMINEL GIGANTELAM

CAREX(SEKT. VIGNEA) NÖ ISEKT. EUCAREX NÜ ROSIRATA SCHL

RUEUS IDAEUS STK POTENTILLA IABERH. FF ERECTA FR EUPATORIUM CANMAR SA MYOSOION AQUATILIS SA STELLARIA ALSINE SA
GRAMINEA SA

POTENTILLA PALUSIRIS SA MENYANTHES TRIF. SA IYPHA LATJFOLIA FR RANUNC. SCELERATUS FR ISEKT. BATRACH. FR LEMNA TRISULCA SA ALISMA PLANT-AQUAT. FR

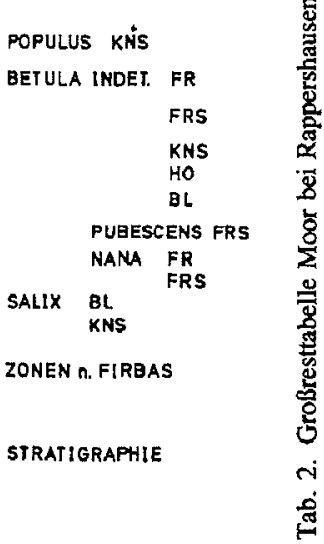


(STREITZ 1984) und „Gaterslebener See“ (MÜLLER 1953) läBt sich die Böllingzeit hier in Ib $\alpha$ und Ib $\beta$ untergliedern. In dem älteren Abschnitt Ib $\alpha$ zeigen Salix, Hippophae und Juniperus Höchstwerte, während sich die Ausbreitung der Birken noch verzögert. Artemisia, Helianthemum und Thalictrum haben bereits ihre Höchstwerte erreicht. Im Abschnitt IbB setzt dann eine verstärkte Birkenausbreitung ein, und die Anteile der Sträucher werden geringer. Ein wichtiges Merkmal der Böllingzeit ist der bei niedrigen Werten gleichmäBige Verlauf der Pinus-Kurve. Ein Anstieg erfolgt erst am Ende des Abschnitts. Für die böllingzeitliche Vegetationsentwicklung zeichnet sich damit folgendes Bild ab: In einer frühen Phase siedeln sich verstärkt Sträucher und kleinere Bestände von (Baum-) Birken an und beenden die tundrenartigen Verhältnisse von Ia. In der jüngeren Phase $(\mathrm{Ib} \beta)$ entstehen erste lichte Birkenwälder, und die Sträucher werden verdrängt. Das Ende der Böllingzeit wird allgemein auf $12300-12100 \mathrm{BP}$ datiert.

Der Klimarückschlag der Älteren Subarktischen Zeit (Zone Ic) ist im Profil Schwarzes Moor E 12 durch 2 Pollenspektren mit einem Anstieg der Gramineen- und dem Rückgang der PinusKurve belegt. Man gewinnt den Eindruck, daß sich dieser Klimarückschlag primär auf die Kiefern-Vorkommen auswirkte. Im Profil Rappershausen (Abb. 7) können die beiden ältesten Spektren lediglich in die Ältere Tundrenzeit i.w.S. gestellt werden. Aufgrund hoher PinusAnteile (ca. $40 \%$ ) kann eine frühe oder mittlere Phase der Böllingzeit hier noch nicht erfaßt sein. Demnach sind beide Spektren in die Endphase von Ib oder nach Ic zu stellen. Zahlreiche Makroreste von Baumbirken machen deutlich, daß Betula in klimatisch begünstigten Landschaften wie dem Grabfeld den Klimarückschlag von Ic überdauert hat. Die Dauer von Ic wird maximal mit 300-350 Jahren angegeben und umfaßt nach BEUG (1976) und OVERBECK (1975) den Zeitraum von 12300-11950 BP. In Rappershausen ergibt eine Extrapolation - aus der ältesten $14 \mathrm{C}$-Datierung und dem Alter der Tuffschicht - für die Grenze V/I a ein Alter von $12000 \mathrm{BP}$.

\subsubsection{Allerödzeit (Zone II)}

Der Übergang zur Allerödzeit ist in keinem der Profile mit einem lithostratigraphischen Wechsel verbunden. Pollenstratigraphisch wird eine Untergliederung der Allerödzeit in eine ältere Birken- und eine jüngere Kiefern-Zeit vorgenommen (II a und IIb). Zone II a zeigt im Schwarzen Moor mit drei Spektren die längste Sequenz; in Rappershausen ist es nur ein Spektrum. Der birkenzeitliche Abschnitt $I$ a ist offenbar nur kurz. Obwohl die Rhön schon relativ weit südlich liegt, nimmt ihre allerödzeitliche Vegetation schon eine Zwischenstellung zwischen den Kieferngebieten Süddeutschlands (z.B. BEUG 1976, LANG 1952) und den reinen Birkengebieten des norddeutschen Flachlandes (z.B. UsiNGER 1982) ein. Das tiefer gelegene Grabfeld folgt aber fast der süddeutschen, rein kiefernzeitlichen Ausbildung der Allerödzeit.

Im Diagramm Schwarzes Moor D $11 \amalg(\mathrm{Abb} .6)$ ergab eine 14C-Datierung für die ältesten Torfe ein Alter von $10995 \pm 100$ BP. Sie haben damit ein ähnliches Alter wie der Laacher Tuff. $\mathrm{Da}$ der Laacher Tuff hier aber nicht erbohrt ist, ergibt sich eine mit den hohen $P$ inus-Anteilen gut übereinstimmende Datienung in dem Abschnitt IIb. In den Profilen Schwarzes Moor E 12 und Rappershausen ist in IIb der Laacher Tuff mit einer Schichtdicke von 7 bzw. $4 \mathrm{~cm}$ nachgewiesen. Als Durchschnittswert zahlreicher Datierungen ermittelt FRECHEN (1959) für die Tuffschicht ein Alter von 11000 BP. Ein höheres Alter nahm OVERBECK (1975) mit 11250 BP an. Für die Vegetationsentwicklung kann zusammenfassend gesagt werden: Mit dem Einsetzen der frühallerödzeitlichen Erwärmung kommt es zu einer raschen Kiefernausbreitung, die in Grabfeld etwas früher als in der Rhön die Vorherrschaft der Birke beendet und die Wälder geschlossener werden läßt. Rasengesellschaften und Sippen wie Hippophae, Juniperus und Helianthemum sind dadurch stark beeinflußt worden. Die Grenze Allerödzeit/Jüngere Tundrenzeit ist aufgrund der vorliegenden Daten schwer zu ermitteln. Durch Interpolation ergibt sich ein Alter von 11000 BP. LANG (1961) zieht diese Grenze bei $10750 \mathrm{BP}$, MANGERUD (1974) schlägt $11000 \mathrm{BP}$ als chronozonale Grenze vor. 


\subsubsection{Jüngere Tundrenzeit (Zone III)}

Die Grenze II/III ist lithostratigraphisch nicht signifikant ausgebildet. Erst in der Mitte bzw. gegen Ende des Abschnitts werden im Schwarzen Moor die Sedimente grobkörniger und reicher an minetalischen Anteilen. In Rappershausen gibt es sogar Braunmoostorfe, die frei von mineralischen Bestandteilen sind. Mit dem Klimarïckschlag der Jüngeren Tundrenzeit kommt es wieder zu einer deutlichen Absenkung der Waldgrenze und zur Wiederausbreitung von Strauchund Rasengesellschaften. Nach LANG (1961) waren die Auswirkungen dieses Stadials wenigstens in Süddeutschland in den Tieflagen (bis $400 \mathrm{~m} \mathrm{NN}$ ) nur gering. Im Untersuchungsgebiet kam es zu einem Rückgang der Pinus-Anteile (erneuter Anstieg gegen Ende des Abschnitts) unter die Werte von IIb, einem Anstieg der Gramineen-Kurve und zu sehr hohen Höchstwerten von Juniperus. Es scheint auch zu einer Auflichtung der (Birken-) Wälder gekommen zu sein. Die Ergebnisse der Großrestanalyse zeigen zwar deutlich, daB Baumbirken (Betula pubescens) in der Rhön diesen Abschnitt überdauerten, aber es kommt im klimatisch begünstigten Gebiet des Grabfeldes zu einer deutlichen Abnahme der Makroreste von Baumbirken (Tab. 2). Gleichzeitig tritt Betula nana auf. Für die Jüngere Tundrenzeit zeichnet sich somit im Untersuchungsgebiet folgendes Bild ab: Mit dem Einsetzen der Klimaverschlechterung werden die allerödzeitlichen Kiefernwälder aufgelichtet, und auch der Bestand an Baumbirken wird reduziert. Erneut breiten sich Betula nana, Juniperus, Salix, Populus, Gramineen, Artemisia, Helianthemum, Thalictrum und Rumex aus. Stärkere minerogene Einlagerungen in die Torfe deuten im Bereich des Schwarzen Moores auf stellenweise offene Böden hin. Gegen Ende der Zone III kommt es dann wieder zu einer Ausbreitung der Kiefer. Die Grenze Spätglazial/Postglazial wird meist auf 10300 BP datiert (2. B. Hammen \& MAarLeVELD 1967, STRAKA 1961). In Rappershausen läßt sich durch Interpolation ebenfalls ein Alter von 10300 BP ermitteln. MANGERUD (1974) schlägt als chronozonales Alter $10000 \mathrm{BP}$ vor.

\subsection{Nacheiszeit}

\subsubsection{Präboreal (Zone IV)}

Das Präboreal wird als Abschnitt mit fortschreitender Erwärmung beschrieben, in dem sich geschlossene Kiefernwälder auch in größeren Höhen ausbreiten (z.B. LANG 1961). Die Diagramme des Untersuchungsgebietes zeigen meist deutlich höhere Pinus-Anteile als in den vorangegangenen Abschnitten. Der Vergleich der beiden Diagramme aus dem Schwarzen Moor zeigt außerdem, daß hier Betula-Gipfel in völlig unterschiedlichen Phasen auftreten und damit den Pollenniederschlag von lokal eng begrenzten Birkenbeständen widerspiegeln. Der erhebliche Rückgang der Gräser deutet darauf hin, daß die Kiefernwälder geschlossener waren als in der Allerödzeit. Spätglazialpflanzen - ausgenommen Artemisia - treten höchstens sporadisch auf. Besonders in IV b häufen sich die Funde von Ulmus und Quercus, so daB jetzt mit ihren Auftreten im Untersuchungsgebiet $\mathrm{zu}$ rechnen ist.

Die Grenze Präboreal/Boreal wurde mit dem Beginn des Steilanstiegs der Hasel-Kurve (Corylus $>10 \%$ ) gezogen. Ihr Alter läßt sich im Schwarzen Moor auf 9050 BP extrapolieren und auf $8900 \mathrm{BP}$ interpolieren, wobei von einer Datierung von $8525 \pm 85 \mathrm{BP}$ im älteren Teil der Zone $V$ ausgegangen wird. Uber das Alter der Grenze IV/V herrscht bei den meisten Autoren eine gute Ubereinstimmung. Bereits FirBas (1949) legte diese Grenze auf 9000 BP; BEHRE (1978) gibt für Mittel- und Nordeuropa dasselbe Alter an.

\subsubsection{Boreal (Zone V)}

Mit der fortschreitenden Erwärmung im Boreal, die bereits über die heutigen klimatischen Verhältnisse hinausgeht (OverbecK 1975), kommt es zur raschen Ausbreitung der Hasel. Im allgemeinen werden im Untersuchungsgebiet Corylus-Maxima von über $100 \%$ erreicht. Durch Interpolation ergibt sch im Schwarzen Moor für den Hasel-Gipfel ein Alter von $7400 \mathrm{BP}$. In 
Rappershausen erscheint eine derartige Berechnung nicht sinnvoll, da eine an der Grenze V/VI gelegene 14C-Datierung mit $8260 \pm 90 \mathrm{BP}$ ein deutlich zu hohes Alter angibt. Zu dem Zeitpunkt des Hasel-Maximums setzt in den Diagrammen ein stärkerer Rückgang der Kiefer ein, mit dem schlieBlich der Abfall der Hasel konform geht. Ulme, Eiche und Linde haben auch hier allmählich Hasel und Kiefer von ihren Standorten verdrängt. In der Rhön wird die Grenze V/VI mit der Überschneidung der Pinus- und EMW-Kurven gezogen. Ebenso verfuhren STECKHAN (1961) im Knüll, WILluTzKI (1962) im Harz und Streitz (1984) im westlichen Rhönvorland. Eine Interpolation liefert hier im Schwarzen Moor ein Alter von nur 7220 BP. MANGERUD (1974) legt diese Grenze dagegen auf $8000 \mathrm{BP}$.

\subsection{3. Älteres Atlantikum (Zone VI)}

Während im westlichen Rhön-Vorland die Pinus-Anteile im gesamten Abschnitt VI bei ca. 10\% liegen (STREITz 1984), findet man in der Rhön zumindest in der älteren Hälfte noch deutlich höhere Werte. Im Grabfeld kommt es erst in der Mitte von VI zur Oberschneidung der EMW- und Pinus-Kurve, und bis zum Ende des Abschnitts sinken die Werte immer noch nicht unter $30 \%$. Dieses weist auf eine Zunahme der Kiefer von Westen nach Osten hin. Für das Untersuchungsgebiet gilt jedoch insgesamt, daß jetzt die Eichenmischwald-Arten zur Vorherrschaft gelangen. Während in den Bergwäldern der Rhön die Ulme dominiert, nimmt in der collinen Stufe des Grabfeldes die Eiche ihre Position ein. Es kommt zu einer ersten Ausbreitung von Fraxinus, Hedera und Viscum. In beiden Diagrammen erfolgt am Ende des Abschnitts ein Anstieg der Fichten-Kurve und schafft für die Grenzziehung eine zeitgleiche Marke. Auch WILLUTZKI (1962) und CHEN (1988) ziehen im Harz und im westlichen Harz-Vorland die Grenze VI/VII an entsprechender Stelle. Im Schwarzen Moor (Abb. 6) ergab eine 14C-Datierung in der Mitte von Zone VI ein Alter von $6130 \pm 75 \mathrm{BP}$. Mit der nächst jüngeren absoluten Datierung läßt sich danach für die Grenze VI/VII ein Alter von $5700 \mathrm{BP}$ interpolieren. Die Fichtenausbreitung scheint hier demnach etwas früher als im Harz erfolgt zu sein. Eine Datierung in Rappershausen ergab für das Ende von Zone VI mit $7555 \pm 70 \mathrm{BP}$ im Vergleich mit der Rhön und benachbarten Gebieten wieder ein zu hohes Alter.

\subsubsection{Jüngeres Atlantikum (Zone VII)}

In der Rhön und im Grabfeld muß das Jüngere Atlantikum als reine EMW-Zeit bezeichnet werden (Mittelwerte knapp 53\%). Die Kiefernkurve sinkt in beiden Diagrammen auf unbedeutende Werte ab. Für die empirische Fagus-Grenze ergab sich im Schwarzen Moor ein Alter von $5,230 \pm 60 \mathrm{BP}$. Obwohl die Fichtenkurve in der Rhön und im Grabfeld Werte bis zu $5 \%$ erreicht, darf man hier nicht von einem Vorkommen der Art ausgehen, sondern muB an Fernflug aus den benachbarten östlichen und südöstlichen Gebieten denken. Anders ist es hingegen im Gebiet der Langen Berge (Moor bei Rottenbach, Abb. 9): Wenn auch hier die stark minerogen durchsetzten Torfe die Zuverlässigkeit der Pollenspektren zweifehaft erscheinen lassen (Picea- und FagusKurve alternieren gemeinsam), beweisen dennoch zahlreiche GroBrestfunde (Nadeln) ein Vorkommen der Fichte, und man muB damit den östlichen Teil des Untersuchungsgebietes dem Fichtenareal zuordnen.

Das Überschreiten der Fagus-Kurve von $1 \%$ (rationelle Grenze) wird in den nördlichen deutschen Landschaften im allgemeinen als Grenze Atlantikum/Subboreal herangezogen. Diese fällt meist auf den Zeitraum um $5000 \mathrm{BP}$, wie folgende Datierungen belegen:

KUBITZKI \& MÜNNICH (1960): $5090 \pm 120 \mathrm{BP}$

Großes Moor, Gifhorn

WILLUTZKI (1962): $5050 \pm 110 \mathrm{BP}$

Auf dem Acker, Oberharz

STrEITZ (1984): $4710 \pm 65$ BP

Wehrdaer Moor 
In der Rhön ist es aufgrund der 14C-Datierungen sinnvoll, die Grenze VII/VIII etwas unterhalb des Fagus-Anstieges, nämlich am Ende des Ulmenabfalls zu ziehen. Hier ergibt sich durch Interpolation ein Alter von 4800 BP. In Rappershausen ist die Grenze VII/VIII durch einen Steilabfall aller EMW-Mitglieder (oberhalb des Spektrums $115 \mathrm{~cm}$ liegt vermutlich ein Hiatus) vorgegeben. Zweifelhaft erscheint die etwas unter diesem Horizont liegende 14C-Datierung, die mit einem Wert von $5610 \pm 125$ BP ca. 1000 Jahre über dem Alter vergleichbarer Spektren aus der Rhön liegt. Immerhin ermöglichen die ansteigenden Kurven von Alnus und Betula in dieser Phase eine Korrelierung mit den Spektren aus dem Schwarzen Moor.

\subsubsection{Subboreal (Zone VIII)}

Dauer und Untergliederung des Subboreals hängen in der Rhön und im Grabfeld von der Ausbreitungsgeschichte der Rotbuche ab. Wie allgemein üblich wurde die Grenze VIIIa/VIIIb bei dem Fagus-Anstieg auf 5\% gezogen. In gleicher Weise verfuhren auch STREITZ (1984), Chen (1988) und STALling (1983). In der Rhön läßt sich für diese Grenze durch Interpolation ein Alter von $3700 \mathrm{BP}$ errechnen, das mit der Datierung WrLluTzKIs von 3700-3400 BP für den Harz ausreichend gut übereinstimmt. Während die Rotbuchen-Ausbreitung in der Rhön in VIIIb zunächst nur langsam voranschreitet und die Dominanz durch einen Steilanstieg am Ende des Abschnitts erreicht wird, ist sie im Grabfeld eher kontinuierlich verlaufen.

In der Rhön setzen in VIII a die Kurven von Carpinus und Abies gleichzeitig ein. Eine 14CDatierung ergibt hier ein Alter von $3940 \pm 65$ BP. Auffälig sind die im gesamten Subboreal auftretenden Schwankungen der Lichthölzer Alnus, Betula und Corylus, die in Rappershausen besonders hohe Maxima erreichen (Abb. 7). Die regionale Waldzusammensetzung wird in Zone VIII jedoch weiterhin von den EMW-Mitgliedern bestimmt, unter denen die Eiche deutlich dominiert. In der Zone VIII a, die in die Schlußphase des Neolithikums fällt, gibt es in der Rhön und im Grabfeld durch die zeitweise geschlossene Plantago lanceolata-Kurve erstmals einen Hinweis auf eine menschliche Besiedlung. In VIIIb ist diese Kurve dann geschlossen, und erste Getreide-PK treten auf. Die Dauer von VIIIb läßt sich für die Rhön auf $3700-2900 \mathrm{BP}$ berechnen, eine Zeitspanne, die sich recht gut mit der Bronzezeit deckt. Die Grenze Subboreal/ Subatlantikum wird allgemein auf den Zeitraum von $2750-2650$ BP gelegt. Gemäß dem Vorgehen in anderen nördlichen Mittelgebirgen erscheint es auch in der Rhön sinnvoll, diese Grenze mit dem Beginn der Fagus-Dominanz zu ziehen. Eine Interpolation ergibt hier ein Alter von $2900 \mathrm{BP}$.

Im Diagramm Rottenbach sind schon zu Beginn des Abschnitts höhere Picea- und FagusWerte zu erkennen, und am Ende von VIII auch ein Anstieg der Abies-Kurve, so daß es noch vor dem Beginn von Zone IX zur Ausbildung von Buchen-Fichten-Tannenwäldern mit eingestreuten Kiefern kommt.

\subsection{6. Älteres Subatlantikum (Zone IX)}

Mit dem Beginn des Älteren Subatlantikums, das in der Rhön und im Grabfeld die uneingeschränkte Vorherrschaft der Rotbuche mit sich bringt, ist die Grundsukzession der Wälder abgeschlossen. Besonders in den montanen Lagen der Rhön kommt es zur Ausbildung von dichten Buchenwäldern, und die im Atlantikum vorherrschenden Lichthölzer werden weitgehend auf Sonderstandorte verdrängt. Während der Abschnitt IX in Rappershausen nur durch vier Pollenspektren erfaßt ist und sich damit ein undifferenziertes Bild der Vegetationsentwicklung ergibt, ist er im Profil aus dem Schwarzen Moor $330 \mathrm{~cm}$ mächtig (Zuwachs des Sphagnum-Torfes ca. 5,4 Jahre $/ \mathrm{cm}$ ). Anhand der Hainbuchenkurve läßt sich Zone IX in die Unterabschnitte IX a und IXb gliedern. Im Schwarzen Moor kommt es zu einem ersten Anstieg der Hainbuchenkurve in der älteren Hälfte von IXa. Dieser Anstieg fällt mit dem Ende einer Phase mit erhöhten Werten der Siedlungsanzeiger und einer kurzzeitig geschlossenen Secale-Kurve zusammen. Eine Extrapolation ergibt an dieser Stelle ein frühlatène-zeitliches Alter von ca. $2300 \mathrm{BP}$. Der Zeitpunkt verstärkter Hainbuchenausbreitung wird meist als Grenze IX a/IXb verwendet. Für die Rhön läßt 
sich nach Angaben OVERBECKs (1962) ein Alter von 1850 BP errechnen. Ihre stärkste Ausbrei tung erfährt die Hainbuche in IXb. In Übereinstimmung mit den Befunden von MOLLER (1953) OVERBECK \& GRIÉZ (1954) und ChEN (1988) muB man mit zwei Carpinus-Gipfeln rechnen, vor denen der ältere unmittelbar oberhalb der Grenze IXa/IX b liegt. Der zweite Gifpel stellt da: Maximum der Kurve dar $(14,1 \%$ im Schwarzen Moor). Im Roten Moor ergibt eine 14C. Datierung nach OvERBECK (1962) an dieser Stelle ein Alter von $560 \pm 120$ u.Z. (nicht kalibriert) Im Harz datiert WILluTZKI (1962) das Carpinus-Maximum auf $600 \pm 50$ u.Z. (nicht kalibriert). Ein Zusammenhang hoher Carpinus-Werte und gleichzeitig stark zurückgehender Siedlungstätig. keit vor dem Hintergnund der Völkerwanderungszeit im 4. -6. Jahrhundert, wie ChEN (1988) unc Stalling (1983) dies belegen, ist im Schwarzen Moor nicht zu erkennen. Die Grenze IX/X wird wie üblich auf den Anstieg der Getreidewerte auf $1 \%$ gelegt. Sie ist außerdem durch einen Anstieg der Betula-Kurve, das Ende der geschlossenen Fraxinus-Kurve, sowic einen Anstieg der Calluna-Anteile charakterisiert. WILLUTZKI (1962) datient diese Grenze im Harz auf $755 \pm 60$ u.Z. (nicht kalibriert), STALLING (1983) im MeiBner auf ca. 900 u.Z. (nicht kalibriert). FIRBAS (1949) verweist darauf, daß das Alter dieser Grenze in Abhängigkeit von der Besiedlung der einzelnen Landschaften zwischen 600 und $1300 \mathrm{u} . \mathrm{Z}$. variieren kann. Anhand der $14 \mathrm{C}$ Datierungen OverBecKs (1962) ergibt sich für die Rhön aufgrund einer Extrapolation ein Alter von ca. 820 u. Z. Damals existierte in unmittelbarer Nähe des Schwarzen Moores bereits eine Siedlung.

In den Langen Bergen dominierten in diesem Abschnitt Fichte und Rotbuche. Die Tannenkurve zeigt nur Werte bis knapp $12 \%$. Ihre geringen Anteile stehen jedoch im Einklang mit Diagrammen aus dem Thüringer Wald (z. B. LANGE 1967).

\subsubsection{Jüngeres Subatlantikum (Zone X)}

Für die Diagramme Rappershausen und Rottenbach erscheint es sinnvoll, eine Untergliederung dieses Abschnitts nach Firbas (1949) in Xa und Xb aufgrund der fortschreitenden PinusAusbreitung vorzunehmen. $X a$ ist stets durch einen starken Rückgang von Buche und Hainbuche charakterisiert. Die Anteile von Pinus und Quercus nehmen zu (Schonung der Eichen als Mastbäume). In Xb kommt es dann zu einer Ausbreitung der Kiefer (Werte bis $80 \%$ ), deren Ursache in der Rodung der Wälder und in ihrer Ausbreitung auf den Mooren zu suchen ist. Einerseits ist die regionale Bedeutung der Kiefer schwer abschätzbar und andererseits dürfte $\mathbf{z}$. B. der Rückgang der Getreideanteile von Xa an in den Diagrammen Rottenbach und Rappershausen nur durch eine sich immer mehr verstärkende Überrepräsentierung der Kiefer in den Pollendiagrammen zu verstehen sein.

Allein das Diagramm Schwarzes Moor D 11 (Abb. 5) ermöglichte wegen seiner Profillänge eine differenzierte Betrachtung siedlungsgeschichtlicher Ereignisse. Es soll deshalb im folgenden näher behandelt werden. Nach OverBeCK (1975) läBt sich der Abschnitt X in Xa, Xb und Xc untergliedern. $X a$ kann als die Zeit der mittelalterlichen Rodungen charakterisiert werden und fält in den Zeitraum von $820-500$ u. $Z$. Xb stellt eine Phase verstärkter Wiesen- und Weidennutzung bis zur zweiten Hälfte des 19. Jahrhunderts dar und $\mathrm{X} c$ schließlich die Zeit der Forste (nach 14C-Datierungen vom Roten Moor: OvERBECK 1962).

Etwa um 1000 u.Z. setzen demnach in der Rhön die ersten großen Rodungen ein und lassen die Anteile der Rotbuche von ca. $55 \%$ auf ca. $20 \%$ im Laufe von 350 Jahren absinken. Es entstehen Äcker und große Wiesen- und Weideflächen. Birke und Hasel finden gute Entwicklungsmöglichkeiten. Die Eiche wird durch ihre Nutzung als Mastbaum gefördert, während Esche, Ulme und Bergahorn wohl in stärkerem Maße für die Laubheufütterung des Viehs genutzt werden und nur in Zeiten rückläufiger Besiedlung als Pollenproduzenten wieder eine Rolle spielen. Hohe Getreidewerte ( $\max .8,6 \%$ ) bis zum Einsetzen der Wüstungsperiode im 14. Jahrhundert belegen, da $B$ die günstigen klimatischen Verhältnisse des Hochmittelalters nicht nur in tieferen und mittleren Lagen der Rhön einen großflächigen Ackerbau ermöglichen. Mit der Klimaverschlechterung im 14. Jahrhundert geht die Besiedlung deutlich zurück, und die Getreidewerte sinken bis auf $2 \%$. In diesen 
Zeitpunkt fällt ein Betula-Maximum. Eine 14C-Datierung (OvERBECK 1962) ergibt $5 \mathrm{~cm}$ unterhalb dieses Spektrums ein Alter von $1360 \pm 50$ u.Z. (nicht kalibriert). Während der 150 Jahre, die bis zu einer erneuten Siedlungsperiode von $1500 \mathrm{u}$. Z. an noch vergehen, kann besonders die Buche viele alte Standorte zurückerobern.

Mit Xb setzt eine emeute Rodungsphase ein, in der die Rhön so stark entwaldet wird, daß der durch Fernflug eingewehte Pollen von Pinus und Picea besonders stark hervortritt. Ein kurzer Anstieg der Fagus-Kurve in der älteren Hälfte von $\mathrm{Xb}$, der mit einem deutlichen Rückgang der Getreidekurve zusammenfällt, könnte die Auswirkungen des Dreißigjährigen Krieges andeuten, in dem die Rhön weitgehend entvölkert wurde. In der jüngeren Phase von Xb beginnen die PinusAnteile in den BP-Spektren zu dominieren und die Getreidekurve erreicht mit über $11 \%$ einen neuen Höchstwert. Die Grenze X $\mathrm{b} / \mathrm{Xc}$ ist durch den weiteren Anstieg der Picea-Kurve und Höchstwerte der Pinus-Anteile definiert, die als Folge intensiver forstlicher Bewirtschaftung der Wälder zustande kommen. Neben der Getreidekurve $(15,1 \%$ ) erreichen auch die Kurven der anderen Siedlungszeiger wie Gramineen, Plantago lanceolata und Rumex jetzt ihre Höchstwerte.

\section{Literatur}

BEHRE, K.-E. (1976): Pollenanalytische Untersuchungen zur Vegetations-und Siedlungsgeschichte bei Flögeln und im Ahlenmoor (Elb-Weser-Winkel). Probleme der Küstenforschung im südlichen Nordseegebiet 11: 101-118.

BEHRE, K.-E. (1978): Die Klimaschwankungen im europäischen Präboreal. Petermanns Geogr. Mitt. 2: 225-246.

BEUG, H.-J. (1957): Untersuchungen zur spätglazialen und frühpostglazialen Floren- und Vegetationsgeschichte einiger Mittelgebirge (Fichtelgebirge, Harz, Rhōn). Flora 145: 167-211.

- (1976): Die spätglaziale und frühpostglaziale Vegetationsgeschichte im Gebiet des ehemaligen Rosenheimer Sees (Oberbayem). Bot. Jahrb. Syst. 95: 373-400.

CнEN, S. (1988): Neue Untersuchungen über die spät- und postglaziale Vegetationsgeschichte im Gebiet zwischen Harz und Leine (BRD). Flora 181: 147-177.

FirBAS, F. (1949/52): Spät- und nacheiszeitliche Waldgeschichte Mitteleuropas nördlich der Alpen I. (1949): Allgemeine Waldgeschichte II. (1952): Waldgeschichte der einzelnen Landschaften. Fischer, Jena.

FreCHEN, J. (1959): Die Tuffe des Laacher Vulkangebietes als quartairgeologische Leitgesteine und Zeitmarken. Fortschr. Geol. Rheinl. u. Westf. 4: 363-370.

GAuHL, F. (1990): Untersuchungen zur Entwicklung des Schwarzen Moores in der Rhön: Verlauf und Ursachen der Vermoorung. Flora 185: 1-16.

GIEs, T. (1972): Vegetation und Ókologie des Schwarzen Moores (Rhön) unter besonderer Berücksichtigung des Kationengehaltes. Dissertationes Botanicae 20.

GUTTENBERG, E. v. (1953/54): Grundzüge der fränkischen Siedlungsgeschichte. Zeitschrift für bayerische Landesgeschichte 17.

HAMMEN, T. VAN DER, \& MAARLEVELD, G. C., u. a. (1967): Stratigraphy, climatic succession and radiocarbon dating of the last Glacial in the Netherlands. Geologie en Mijnbouw 46: 79-95.

HILKE, H. (1970): Die Grab- und Hortfunde der Umenfelderkultur aus Ober- und Mittelfranken. Materialhefte zur bayerischen Vorgeschichte 23.

KNOCH, K. (1952): Klimaatlas von Bayern. Bad Kissingen.

KossacK, G. (1970): Gräberfelder der Hallstattzeit an Main und Fränkischer Saale. Materialhefte zur bayerischen Vorgeschichte 24.

KUBITZKI, K., \& MÜNNICH, K. O. (1960): Neue C14-Datierungen zur nacheiszeitlichen Waldgeschichte Nordwestdeutschlands. Ber. Deutsch. Bot. Ges. 73: 137-146.

LANG, G. (1952): Zur späteiszeitlichen Vegetations- und Florengeschichte Südwestdeutschlands. Flora 139: 243-294.

- (1961): Die spät- und frühpostglaziale Vegetationsentwicklung im Umkreis der Alpen. Eiszeitalter und Gegenwart 12: $9-17$.

LANGE, E. (1967): Zur Vegetationsgeschichte des Beerberggebietes in Thüringer Wald. Feddes Repert. 76: 205-219.

MALTER, W. (1965): Oberfranken-West. Deutsche Landeskunde. Nürnberg.

- (1967): Oberfranken-Ost. Deutsche Landeskunde. Nürnberg.

MANGERUD, J. U. a. (1974): Quatemary stratigraphy of Norden, a proposal for terminology and classification. Boreas 3: $109-126$. 
Mensching, H. (1957): Geomorphologie der Hohen Rhön und ihres südlichen Vorlankes. Festschrift zum ? Deutschen Geographentag Würzburg 1957. Würzburger geographische Arbeilen $4 \mathbf{5}$.

MÚlefr, H. (1953): Zur späl- und nacheiszeitlichen Vegetationsgeschichie des mitteldeutschen Trockengebiet: Nova Acta Leopoldina 16 (110): 3-67.

MULleR-KARPE, H. (1959): Beiträge zur Chronologie der Umenfelderzeit nördlich und sudich der Alpen. Römisc germanische Forschungen, Berlin 22.

OVERBECK, F. (1928): Studien zur postglazialen Waldgeschichte der Rhōn. Z. Bot. 20: 145-206.

- (1962): Einige Hinweise zu den Exkursionen im nordwestdeutschen Flachland und in der Rhơn. $V$. Inten Symposium der Quartärbotaniker in Kiel und Göttingen, Universitāt Kiel.

- (1975): Botanisch-Geologische Moorkunde. Neumanster.

- \&GRiEz, I. (1954): Mooruntersuchungen zur Rekurrenzflächenfrage und Siedlungsgeschichie der Rhön. Flora 141 $51-99$.

ReIMERs, H. (1924): Die Vegetation der Rhönmoore. Repert. Spec. Nov. Regni Vegetabilis, Beiheft 26: 21 -55. SEIBERT, P. (1968): Übersichtskarte der natürlichen Vegetations gebiete von Bayem 1 :500000 mit Erlăuterungen. Bat Godesberg.

STALLING, H. (1983): Untersuchungen zur nacheiszeitlichen Vegetationsgeschichte des MeiBners (Nordhessen). For: 174: $357-376$.

STzскнAN, H.-U. (1961): Pollenanalytisch-vegetationsgeschichtliche Untersuchungen zur frühen Siedlungsgeschichte im Vogelsberg, Knüll und Solling. Flora 150: 514-551.

STRAKA, H. (1961): Relative und absolute Datierung quartărer Ablagenungen. Die Naturwissenschaften 48 : $324-332$.

STREITz, B. (1984): Vegetationsgeschichtliche Untersuchungen an zwei Mooren osthessischer Subrosionssenken. Beitr. Naturk. Osthessen, Fulda 20: 3-77.

Stuhlfauth, A. (1962): Vorgeschichte Oberfrankens: Von der mittleren Steinzeit bis zur Bronzezeit. Bayreuth.

USINGER, H. (1982): Zur vegetations- und klimageschichtlichen Gliederung des Alleröds nach Untersuchungen in Blixmoor (Schleswig-Holstein). Schr. Naturw. Verl. Schleswig-Holstein, Kiel 52: 29-45.

VOLLRATH, H. (1957): Die Pflanzenwelt des Fichtelgebirges und benachbarter Landschaften in geobotanischer Schau. (Geobotanik des Fichtelgebirges und benachbarter Florenbezirke). Ber. Naturwiss. Gesell. Bayreuth 9: 5-250.

Vonderau, J. (1926): Die Besiedlung der Rhön in vor- und frügeschichtlicher Zeit. Festschrift zum 50 jährigen Jubiläums des Rhönclubs. Fulda.

WilluTZkı, H. (1962): Zur Waldgeschichte und Vermoorung sowie über Rekurrenzflächen im Oberharz. Nova Acta Leopoldina N.F. 25 160: 3-52.

Eingegangen am 21. August 1989

Anschrift des Autors: Dipl.-Biol. JÜrgeN HAHNe, Institut für Palynologie und Quartairwissenschaften der Universität, Wilhelm-Weber-Straße 2, W-3400 Göttingen, BRD. 